\title{
Kinematics of nearby subdwarf stars
}

\author{
M. I. Arifyanto, B. Fuchs, H. Jahreiß, and R. Wielen
}

\author{
Astronomisches Rechen-Institut, Mönchhofstraße 12-14, 69120 Heidelberg, Germany \\ e-mail: arifyanto@ari.uni-heidelberg.de
}

Received 9 December 2003 / Accepted 10 December 2004

\begin{abstract}
We present an analysis of the space motions of 742 subdwarf stars based on the sample of Carney et al. (1994, AJ, 107, 2240). Hipparcos parallaxes, TYC2+HIP proper motions and Tycho 2 proper motions were combined with radial velocities and metallicities from CLLA. The kinematical behavior is discussed in particular in relation to their metallicities. The majority of these sample stars have metal abundances of $[\mathrm{Fe} / \mathrm{H}]>-1$ and represent the thick disk population. The halo component, with $[\mathrm{Fe} / \mathrm{H}]<-1.6$, is characterized by a low mean rotation velocity and a radially elongated velocity ellipsoid. In the intermediate metallicity range $(-1.6 \leq[\mathrm{Fe} / \mathrm{H}] \leq-1)$, we find a significant number of subdwarfs with disklike kinematics. We interpret this population of stars as a metal-weak thick disk (MWTD) population.
\end{abstract}

Key words. stars: subdwarfs - Galaxy: kinematics and dynamics - Galaxy: solar neighbourhood

\section{Introduction}

Studies of the kinematics of various stellar populations in the Galaxy, in particular the thick disk and the halo, have long been limited by the availability of large samples of stars with measurements of proper motions, radial velocities, distances, and metallicities. Such data are required in order to constrain plausible scenarios for the formation and evolution of the Milky Way. Samples of nearby subdwarf stars with high space motions provide an observationally convenient probe of the structure of the Galaxy. The large, proper-motion-selected, stellar samples of Carney et al. (1994, hereafter CLLA) have proved particularly valuable for studying the kinematics and chemical abundances within a few kiloparsecs of the Sun.

The correlation between kinematics and metallicity gives useful information for formulating theories of galactic structure. Differences in chemistry and space velocities are crucial in defining the different populations within the Galaxy and inferring their origins. Relevant studies of the kinematical behavior of stars, in particular in relation to their metallicities, were presented by e.g. Morrison et al. (1990, hereafter MFF) using a sample of K giants whose metallicities are measured using the DDO photometric system, Nissen \& Schuster (1991) using late $\mathrm{F}$ and $\mathrm{G}$ dwarfs and subgiants, Chiba \& Yoshii (1998) using red giants and RR Lyrae stars, Martin \& Morrison (1998) with a sample of nearby RR Lyrae stars and Chiba \& Beers (2000) using 1203 metal-poor solar-neighborhood stars.

The Galactic halo is characterized by a roughly spherical space distribution with close to zero net rotation. Its stars are metal poor, with a peak metallicity at $[\mathrm{Fe} / \mathrm{H}]=-1.6$ (Laird et al. 1988). The halo population in the solar neighborhood is not purely a relic of a monolithic, "rapid" collapse (Carney et al. 1996). There have been several suggestions of a twocomponent halo, with a flattened component in the inner halo and a more spherical outer halo (Sommer-Larsen \& Zhen 1990; Carney et al. 1996).

The Galactic thick disk is the kinematically hottest portion of the disk of the Galaxy, with a scale height of 1.0 to $1.5 \mathrm{kpc}$ and rotates with a velocity of about $170 \mathrm{~km} \mathrm{~s}^{-1}$ (Gilmore et al.1989). The thick disk is usually considered to be dominated by stars in the range $[\mathrm{Fe} / \mathrm{H}]>-1$ (Freeman 1987), peaking at about $[\mathrm{Fe} / \mathrm{H}]=-0.5$ (Carney et al. 1989). Many workers have claimed the existence of a metal weak tail of the thick disk component in the range $-1.6 \leq[\mathrm{Fe} / \mathrm{H}] \leq-1$ (MFF; Beers \& Sommer-Larsen 1995; Chiba \& Beers 2000). MFF found a fraction of $72 \%$ of the stars in this metallicity range in a "metal-weak thick disk" (MWTD), rotating rapidly at $V_{\text {rot }} \approx 170 \mathrm{~km} \mathrm{~s}^{-1}$. Another large fraction of MWTD was found also by Beers \& Sommer-Larsen (1995). Their MWTD, rotating at $V_{\text {rot }} \approx 195 \mathrm{~km} \mathrm{~s}^{-1}$, accounts for about $60 \%$ of the stars in the range $-1.6 \leq[\mathrm{Fe} / \mathrm{H}] \leq-1$ in the solar neighborhood, and it possesses an extremely metal-weak tail down to $[\mathrm{Fe} / \mathrm{H}] \leq-2$. Chiba \& Beers (2000) estimated the fraction of MWTD at about $30 \%$ of the metal-poor stars in the abundance range $-1.7 \leq[\mathrm{Fe} / \mathrm{H}] \leq-1$, which is smaller than the fraction derived by MFF and Beers \& Sommer-Larsen (1995), but larger by $\sim 10 \%$ than the result of Chiba \& Yoshii (1998) using solar neighborhood red giants and RR Lyrae stars.

The investigation of thick-disk and halo kinematics may only be applicable to a specific place in the Galaxy and may have fine structure of the velocity distribution smoothed out by the velocity resolution of the study (Martin \& Morrison 1998). Here, we study the kinematics of solar neighborhood subdwarf 
stars based on the sample of high proper motion stars by CLLA. CLLA have measured photometric parallaxes, radial velocities, and metallicities of mainly A to early G stars, many late $\mathrm{G}$ and some early K stars in the Lowell Proper Motion Catalogue. In total the CLLA sample contains 1464 stars. In their paper there are listed 1269 stars with kinematical parameters and 1261 stars with metallicity parameters, and there are 1447 stars with radial velocities in their catalog. The radial velocity precision of their sample lies in the range of 0.4 to $1.3 \mathrm{~km} \mathrm{~s}^{-1}$. About $15 \%$ of their sample are binaries or multiple systems. The typical accuracy of the metallicities was estimated to be \pm 0.13 dex.

The photometric parallax of CLLA was replaced in our study by using the high precision parallax of Hipparcos catalogue. We used the Astrometric Catalog TYC2+HIP (Wielen et al. 2001) for the proper motions of stars with Hipparcos parallaxes. This catalogue is derived from a combination of the Hipparcos Catalogue with proper motions given in the Tycho-2 catalogue with direct solutions (Wielen et al. 2001) and previous earthbound measurements. We still use the high precision radial velocities and metallicities of the CLLA catalogue for our study. Previous work using Hipparcos subdwarfs was done by Reid (1998) and Fuchs et al. (1998; hereafter FJW). In this previous work we discussed the kinematical behaviour of the 560 subdwarfs for which improved parallaxes and proper motions were obtained by Hipparcos, in relation to their metallicities. In the present paper we increase the size of the sample considerably by applying a correction to the photometric CLLA distances determined using stars with Hipparcos parallaxes.

In Sect. 2 we describe the selection of the sample and the photometric correction. In the next section we determine the kinematical properties, i.e. the $3 \mathrm{D}$ velocities with respect to the Sun, and discuss the kinematical behavior of the subdwarfs, in particular in relation to their metallicities. Our conclusions are summarized in Sect. 4.

\section{Data}

The CLLA data set of 1447 stars has been cross-identified with the Astrometric Catalogue TYC2+HIP (Wielen et al. 2001) and we found 545 stars in common. About 700 CLLA stars did not appear in the TYC $2+$ HIP catalogue, but were then crossidentified with the Tycho-2 Catalogue (Høg et al. 2000), and we found 259 subdwarfs with Tycho- 2 proper motions. The proper motion accuracy of Tycho 2, derived from a comparison with the Astrographic Catalogue and 143 other ground-based astro-

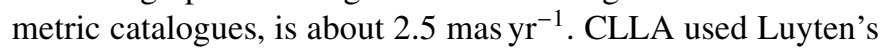
NLTT proper motions for the calculation of the space velocity components. These proper motions have typical errors of 20 to 25 mas yr$^{-1}$. Therefore, TYC2+HIP and Tycho- 2 proper motions provide an enormous improvement in the accuracy of the tangential velocities.

\subsection{Color magnitude diagram}

Figure 1 shows the color magnitude diagram for all 545 identified CLLA stars. The absolute magnitudes and their standard

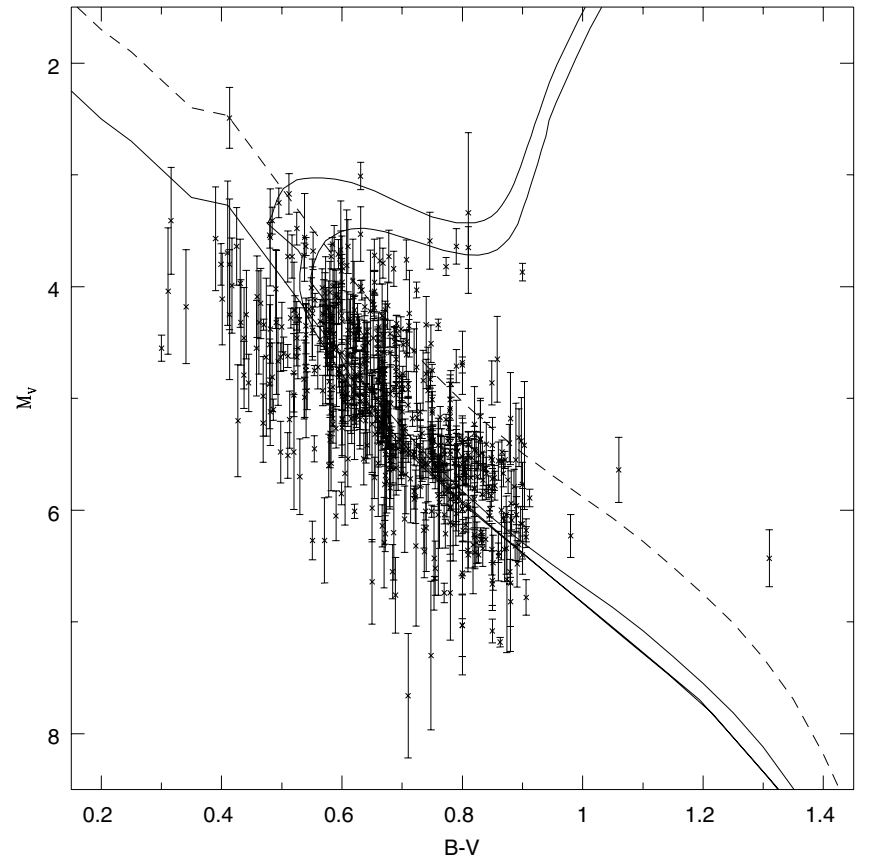

Fig. 1. Color-magnitude diagram for all identified CLLA stars. Hipparcos parallaxes were used to determine $M_{V}$ and its standard error. The full lines indicate the zero age main sequence (ZAMS) and main sequences of the old open clusters M 67 and NGC 188. The dashed line is the ZAMS shifted upward by $\Delta M_{V}=0.8 \mathrm{mag}$, used to remove the contamination by subgiants and giants.

errors are based on the Hipparcos parallaxes and their errors. The $B-V$ colors were taken from the Hipparcos catalogue. Some stars for which no distance was given were already recognized by CLLA as subgiants. However, we can see clearly from the CM-diagram in Fig. 1 that there is still contamination by previously undetected subgiants and giants. To avoid these, we have removed all stars lying above a line in the CM-diagram defined by the zero age main sequence of stars with solar metallicity shifted upward by $\Delta M_{V}=0.8 \mathrm{mag}$. About $8 \%$ contaminating stars were eliminated in this way.

\subsection{Test of photometric distances}

We determined the overall correction of the photometric distance scale of CLLA by analyzing the parallax difference. There are 539 CLLA stars which have both photometric and trigonometric parallaxes in our sample (CLLA-TYC2+HIP). We compared the Hipparcos parallax with the photometric parallax of CLLA (as shown in Fig. 2). The error bars represent both Hipparcos and CLLA parallax errors. A typical error in the absolute magnitude of CLLA stars of $\sigma_{M_{V}}=0.3 \mathrm{mag}$ is assumed. We used a least $\chi^{2}$ method applicable when the data have errors in both coordinates. The $\chi^{2}$-function is chosen according to Press et al. (1992)

$\chi^{2}=\sum_{i=1}^{N} \frac{\left(y_{i}-b x_{i}\right)^{2}}{\sigma_{y_{i}}^{2}+b^{2} \sigma_{y_{i}}^{2}}$.

The slope of the regression $b$ derived from our 539 subdwarfs, is $b=1.116 \pm 0.008$. For comparison, a sample with 

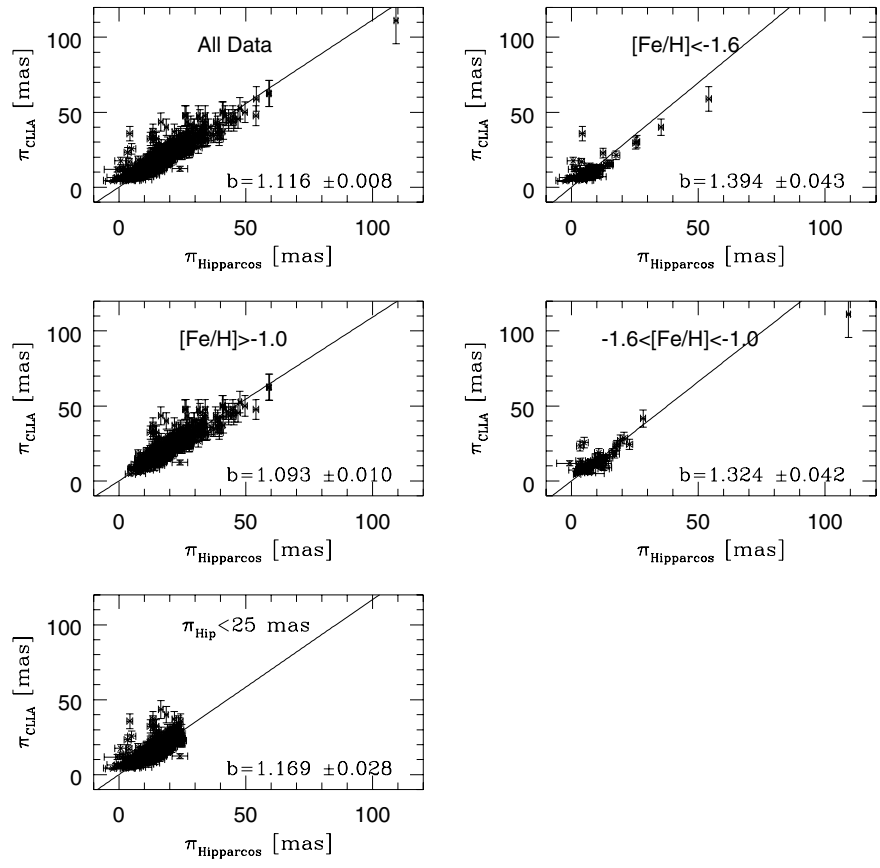

Fig. 2. Hipparcos trigonometric parallaxes versus the photometric parallaxes of CLLA, for 539 stars (top-left) and for different metallicity cuts : $[\mathrm{Fe} / \mathrm{H}]<-1.6$ (top-right), $[\mathrm{Fe} / \mathrm{H}]>-1$ (center-left), $-1.6 \leq[\mathrm{Fe} / \mathrm{H}] \leq-1$ (center-right), and for stars at large distances, $\pi_{\text {Hip }}<25$ mas (bottom-left). The full line is a linear fit to the data.

$\pi_{\text {Hip }} \leq 25$ mas leads to a larger correction of $b=1.169 \pm 0.028$. We also tried cuts in the metallicties, with the result that for more metal-poor stars a larger correction was needed. For stars with $[\mathrm{Fe} / \mathrm{H}]>-1$, which are dominated by thick disk stars, $-1.6 \leq[\mathrm{Fe} / \mathrm{H}] \leq-1$, and extreme metal-poor stars with $[\mathrm{Fe} / \mathrm{H}]<-1.6$ we find slopes of the regression line $b=$ $1.093 \pm 0.010, b=1.324 \pm 0.042$ and $b=1.394 \pm 0.043$, respectively. Jahreiß et al. (1997) and FJW have found similar corrections using a smaller sample of subdwarfs. The data points at the upper right corner of the first plot (All Data) and the fourth plot $(-1.6 \leq[\mathrm{Fe} / \mathrm{H}] \leq-1)$ represent the star HIP 57939, which is the nearest star in our sample with $\pi_{\text {Hip }}=109$ mas. No significant changes in the slopes of both plots (less than $1 \sigma)$ are found, if we omit HIP 57939 when calculating the slopes.

All these corrections are used to calibrate CLLA photometric parallaxes in our sample with no Hipparcos parallaxes and about 35 stars with low accuracy Hipparcos parallaxes i.e. $\pi_{\text {Hip }}<5$ mas and $\pi_{\text {Hip }} / \sigma_{\pi_{\text {Hip }}}<3$ mas.

\section{Kinematical properties}

Using the parallaxes supplied by the Hipparcos catalogue, proper motions by the TYC2+HIP and Tycho- 2 catalogue, and radial velocities given in CLLA, the space velocity components $U, V$, and $W$, which are directed to the Galactic center, direction of galactic rotation, and north galactic pole, respectively, have been calculated with respect to the Sun and then reduced to the LSR (local standard of rest). For the latter Delhaye's (1965) values $+9,+12,+7 \mathrm{~km} \mathrm{~s}^{-1}$ were adopted for $U_{\odot}, V_{\odot}, W_{\odot}$, respectively. Finally, the velocity components were transformed onto a frame rotating with circular velocity $V_{\text {circ }}=-220 \mathrm{~km} \mathrm{~s}^{-1}$ relative to the LSR, i.e. the expected rest frame of our Galaxy (e.g. Wielen 1986). The rotational velocity is defined as $V_{\text {rot }}=V-V_{\text {circ }}$.

Figure 4 shows the $U, V_{\text {rot }}$, and $W$ velocities of the samples CLLA-TYC2+HIP and CLLA-Tycho 2 as scatter plots. The $U$-distribution indicates that the present sample was kinematically selected. The CLLA Catalog is based on a proper motion catalog so that stars with small tangential velocities are missing. We can see clearly that for small $U$-values the diagrams are sparsely populated. This is also seen in the $V$-velocities. The stars with metallicities $[\mathrm{Fe} / \mathrm{H}]>-1$ lag on the average by about $40 \mathrm{~km} \mathrm{~s}^{-1}$, i.e. are thick disk stars. The old thin disk stars are missing (cf. also Fig. 5). In the $W$-velocities no kinematical bias is visible; it is apparently lost in projection. However, since we are mainly interested in the kinematics of the halo stars, this bias is of no consequence in the present context. It is evident from this figure that metal-poor stars with $[\mathrm{Fe} / \mathrm{H}]<-1$ have larger random motions compared with metal-rich ones $[\mathrm{Fe} / \mathrm{H}]>-1$. This shows that the kinematic properties change rather abruptly at $[\mathrm{Fe} / \mathrm{H}] \approx-1$ to -2 , which is probable the transition region from halo to disk component (Ryan \& Norris 1991; Chiba \& Yoshii 1998).

The mean motion with respect to the LSR and velocity dispersions were calculated for different groups in $[\mathrm{Fe} / \mathrm{H}]$. The results are presented in Table 1 . The most metal-deficient stars in the samples, more metal poor than $[\mathrm{Fe} / \mathrm{H}]=-1.6$, are dominated by members of the halo population. These stars exhibit a radially elongated velocity ellipsoid $\left(\sigma_{U}, \sigma_{V}, \sigma_{W}\right)=$ $(189 \pm 13,97 \pm 7,98 \pm 7)$ and $(157 \pm 12,87 \pm 7,77 \pm 6) \mathrm{km} \mathrm{s}^{-1}$ and show no net rotation, $\left\langle V_{\text {rot }}\right\rangle=1 \pm 13$ and $15 \pm 14 \mathrm{~km} \mathrm{~s}^{-1}$ for the CLLA-TYC2+HIP and CLLA-Tycho 2 samples, respectively, which are in good agreement with RR Lyrae kinematics of Martin \& Morrison (1998) and Layden et al. (1996). Chiba \& Beers (2000) found a lower velocity dispersion in the $U$-direction, $\left(\sigma_{U}, \sigma_{V}, \sigma_{W}\right)=(141 \pm 11,106 \pm 9,94 \pm 8) \mathrm{km} \mathrm{s}^{-1}$ from their 1203 non-kinematically selected stars.

The velocity dispersion components of the sample in the more metal-rich abundance ranges decrease as the contribution of the thick disk component progressively increases. In particular, for $[\mathrm{Fe} / \mathrm{H}]>-1.0$ the contribution of the halo component is expected to be negligible. Our CLLA+TYC2+HIP sample in this metallicity range has velocity dispersions $\left(\sigma_{U}, \sigma_{V}, \sigma_{W}\right)=$ $(74 \pm 2,50 \pm 1,37 \pm 1)$ with $V_{\text {rot }}=176 \mathrm{~km} \mathrm{~s}^{-1}$, which is in agreement with thick disk samples of Martin \& Morrison (1998) of RR Lyrae stars and Chiba \& Beers (2000) of solarneighborhood stars.

Our CLLA-Tycho 2 sample is more sparsely distributed than the CLLA-TYC2+HIP sample in the metallicity range $[\mathrm{Fe} / \mathrm{H}]>-1$. To understand this, we note that the CLLATycho 2 sample was drawn from the CLLA stars that are not in the Hipparcos catalogue. This might imply that mainly CLLA stars with magnitudes brighter than 10.5 mag fall in our CLLA-TYC2+HIP sample and stars with magnitudes fainter than $10.5 \mathrm{mag}$ are in the CLLA-Tycho 2 sample. Stars with fainter apparent magnitudes are at larger distances and velocities compared to the Hipparcos stars. The minimum distances 
Table 1. Mean velocities and velocity dispersion of the sample stars.

\begin{tabular}{lrcccccc}
\hline \hline dex & $N$ & $\langle U\rangle$ & $\langle V\rangle$ & $\begin{array}{c}\langle W\rangle \\
\mathrm{km} \mathrm{s}^{-1}\end{array}$ & $\sigma_{U}$ & $\sigma_{V}$ & $\sigma_{W}$ \\
\hline CLLA-TYC2+HIP & & & & & & & \\
{$[\mathrm{Fe} / \mathrm{H}]>-1.0$} & 381 & $-11 \pm 4$ & $-44 \pm 3$ & $-4 \pm 2$ & $74 \pm 2$ & $50 \pm 1$ & $37 \pm 1$ \\
$-1.6 \leq[\mathrm{Fe} / \mathrm{H}] \leq-1.0$ & 49 & $-35 \pm 23$ & $-163 \pm 12$ & $-1 \pm 11$ & $158 \pm 11$ & $85 \pm 6$ & $74 \pm 5$ \\
{$[\mathrm{Fe} / \mathrm{H}]<-1.6$} & 53 & $-5 \pm 26$ & $-219 \pm 13$ & $-1 \pm 13$ & $189 \pm 13$ & $97 \pm 7$ & $98 \pm 7$ \\
\hline CLLA-Tycho2 & & & & & & & \\
{$[\mathrm{Fe} / \mathrm{H}]>-1.0$} & 169 & $-9 \pm 8$ & $-91 \pm 6$ & $-5 \pm 4$ & $110 \pm 4$ & $81 \pm 3$ & $58 \pm 2$ \\
$-1.6 \leq[\mathrm{Fe} / \mathrm{H}] \leq-1.0$ & 50 & $51 \pm 17$ & $-180 \pm 10$ & $4 \pm 9$ & $121 \pm 9$ & $68 \pm 5$ & $61 \pm 4$ \\
{$[\mathrm{Fe} / \mathrm{H}]<-1.6$} & 40 & $36 \pm 25$ & $-205 \pm 14$ & $-19 \pm 12$ & $157 \pm 12$ & $87 \pm 7$ & $77 \pm 6$ \\
\hline
\end{tabular}
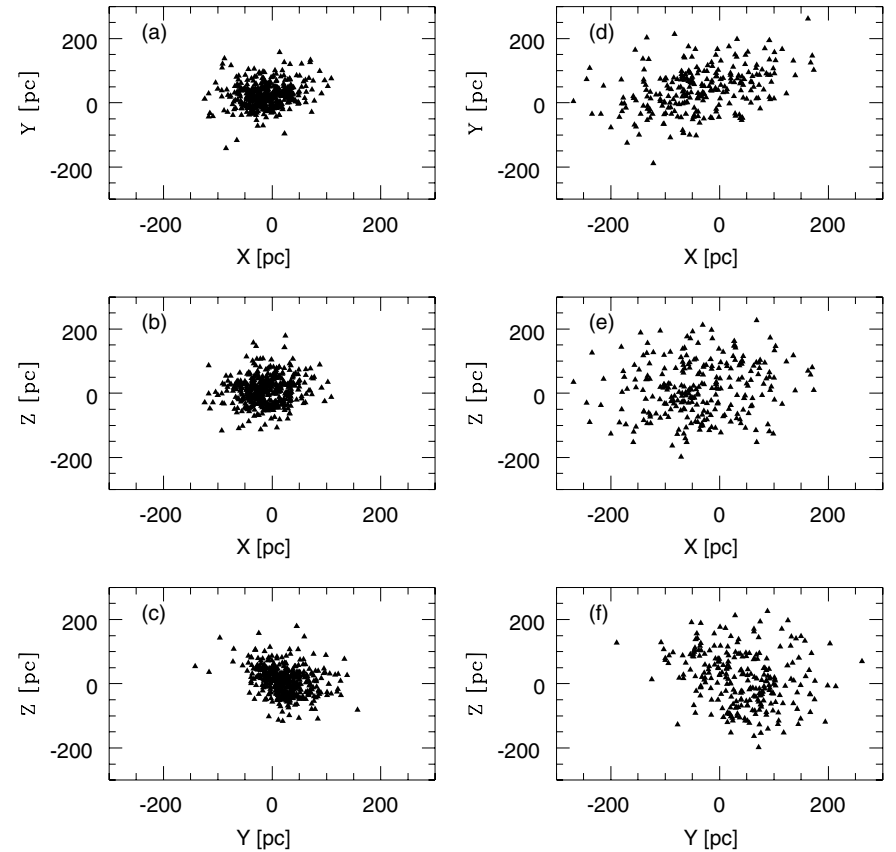

Fig. 3. Spatial distribution of the samples CLLA-TYC2+HIP a) to c) and CLLA-Tycho-2 d) to $\mathbf{f}$ ), respectively. $X$ points towards the Galactic center, $Y$ in the direction of galactic rotation and $Z$ towards the Galactic north pole.

for each sample are 17 and 40 pc for CLLA-TYC2+HIP and CLLA-Tycho 2, respectively. Figure 3, where we plot the spatial distributions in $X, Y$ and $Z$ shows this clearly. We can find the minimum tangential velocity using the minimum distances and mean proper motions for both samples, using

$V_{T \min }=4.74 \frac{\langle\mu\rangle}{1000} d_{\min }$

where $\langle\mu\rangle, d_{\min }$, and $V_{T \text { min }}$ denote mean proper motions in mas $\mathrm{yr}^{-1}$, minimum distances in parsecs and minimum tangential velocities in $\mathrm{km} \mathrm{s}^{-1}$ for each subdwarf sample. We found for the CLLA-Tycho 2 sample $V_{T \text { min }}>50 \mathrm{~km} \mathrm{~s}^{-1}$, which might explain why there are comparatively few thick disk stars in this sample (cf. Fig. 5).
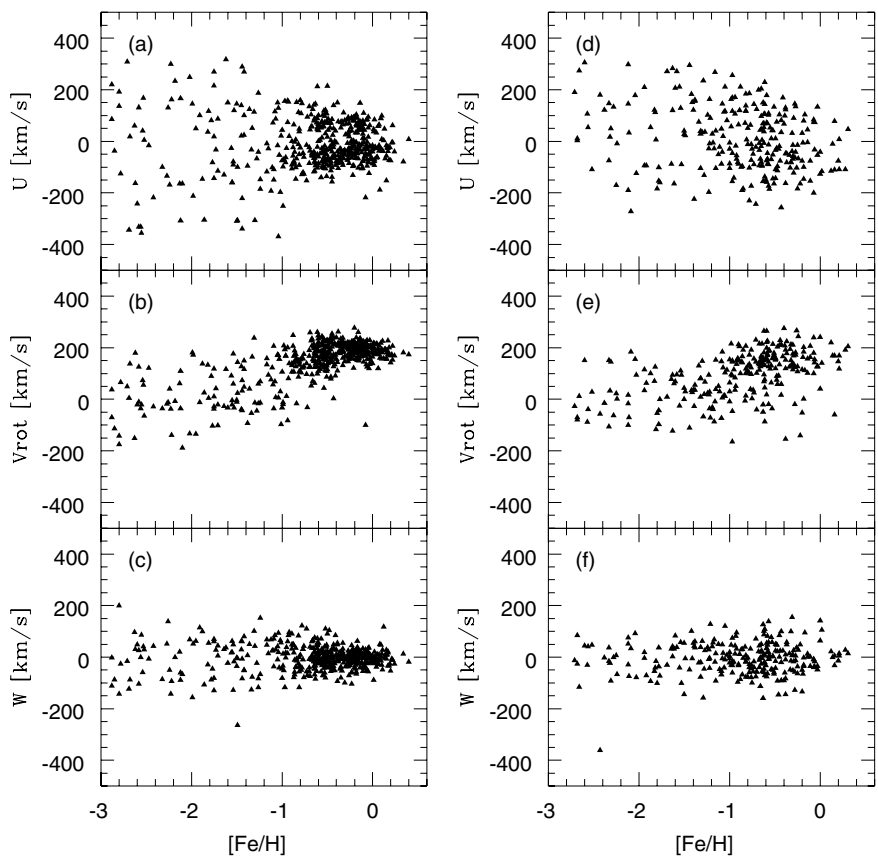

Fig. 4. Space velocity components $\left(U, V_{\text {rot }}, W\right)$ versus metallicity $[\mathrm{Fe} / \mathrm{H}]$ of the samples CLLA-TYC2+HIP a) to c) and CLLA-Tycho-2 d) to f), respectively.

\section{1. $V_{\text {rot }}$ distributions of subdwarfs}

The corresponding $V_{\text {rot }}$-velocity distributions for the samples CLLA-TYC2+HIP and CLLA-Tycho 2 are shown in Figs. 6 and 7 , respectively. The first group, $[\mathrm{Fe} / \mathrm{H}]>-1 \mathrm{dex}$, represents what are obviously the thick disk stars. The third group, $[\mathrm{Fe} / \mathrm{H}]<-1.6$ dex, consists of extreme metal-poor stars, dominated by members of the halo population. The histograms of the samples CLLA-TYC2+HIP and CLLA-Tycho 2 can be fitted by Gaussian distributions.

The second group, $-1.6 \leq[\mathrm{Fe} / \mathrm{H}] \leq-1$ dex, shows a peculiar kinematics. A Kolmogorov-Smirnov test, which avoids binning of the data, shows that the velocity distribution of the very metal-poor stars, $[\mathrm{Fe} / \mathrm{H}] \leq-1.6$, in the combined sample is statistically different from the velocity distribution of the intermediate population, $-1.6 \leq[\mathrm{Fe} / \mathrm{H}] \leq-1.0$. The maximum deviation of the normalized cumulative distribution between the two groups is $D=0.241$ and thus significantly larger than the critical value $D_{0.05}=0.196$ (Sachs 1988), which leads to a 


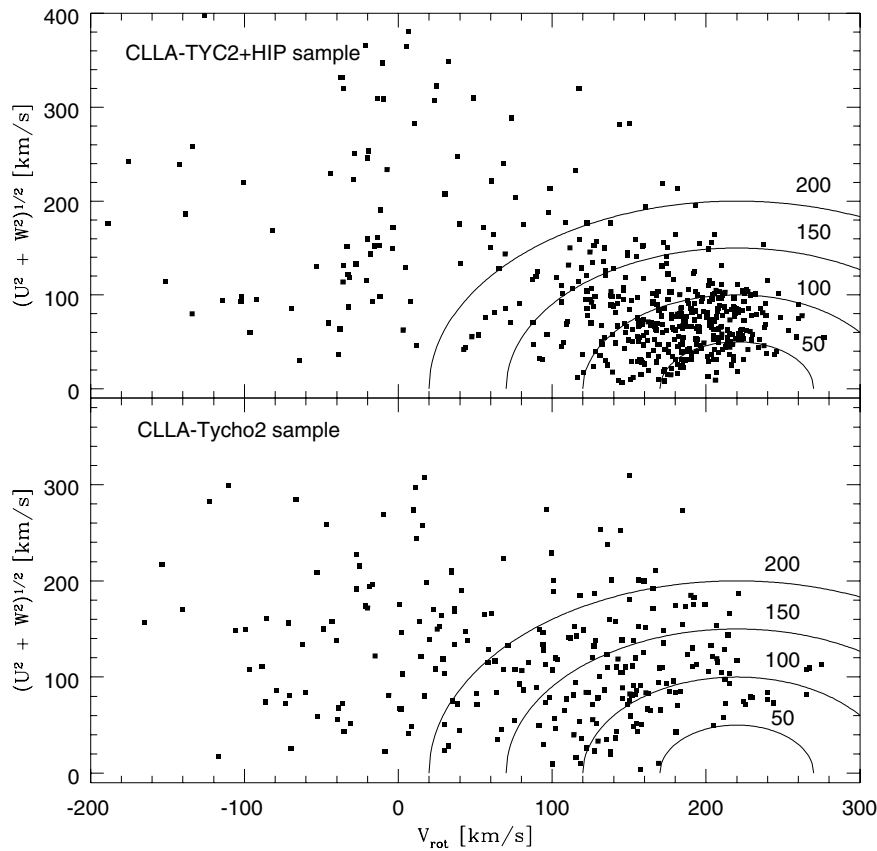

Fig. 5. Toomre diagram: $\left(U^{2}+W^{2}\right)^{1 / 2}$ versus $V_{\text {rot }}$ distributions of CLLA-TYC2+HIP and CLLA-Tycho 2 samples. The solid lines represent total velocities of 50,100, 150 and $200 \mathrm{~km} \mathrm{~s}^{-1}$, respectively.
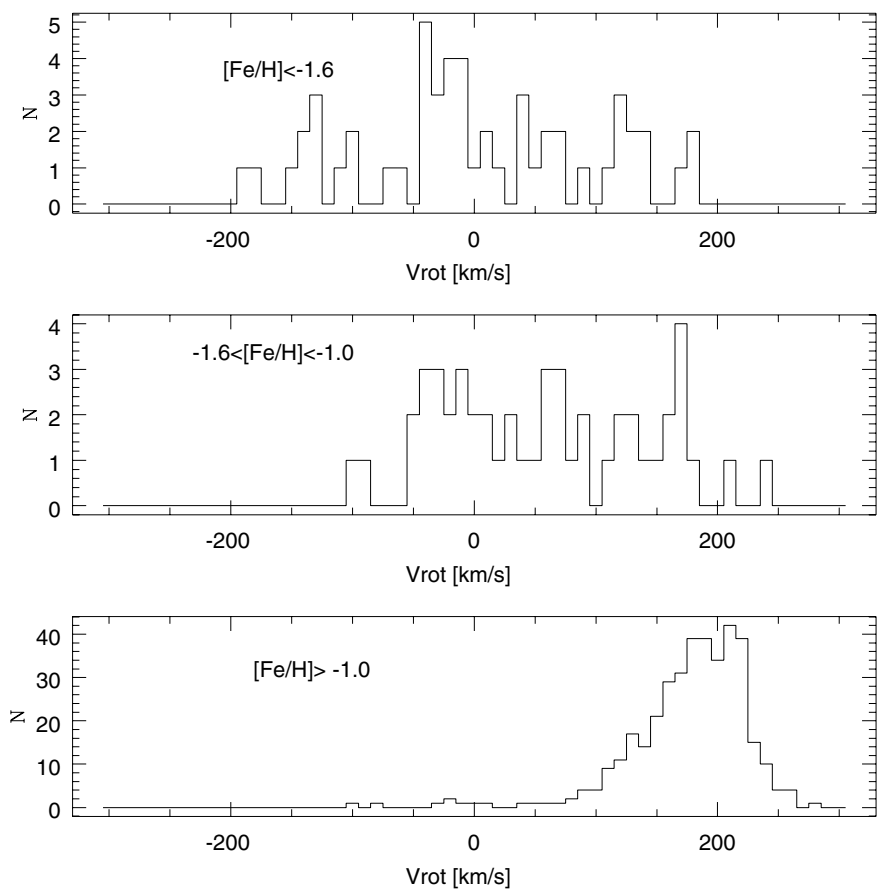

Fig. 6. Rotational velocity $\left(V_{\text {rot }}\right)$ distributions of sample CLLATYC2+HIP grouped according to their metallicities. The velocities are reduced to the local standard of rest.

rejection of the hypothesis of the statistical similarity of the velocity distributions of the two groups. Similarly we have shown that the velocity distribution of the metal-poor stars is symmetric with respect to $V_{\text {rot }}=0 \mathrm{~km} \mathrm{~s}^{-1}\left(D=0.149, D_{0.05}=0.282\right)$, whereas the velocity distribution of the intermediate group is asymmetric $\left(D=0.375, D_{0.05}=0.300\right)$. Thus the intermediate group seems to represent a different population of halo stars.
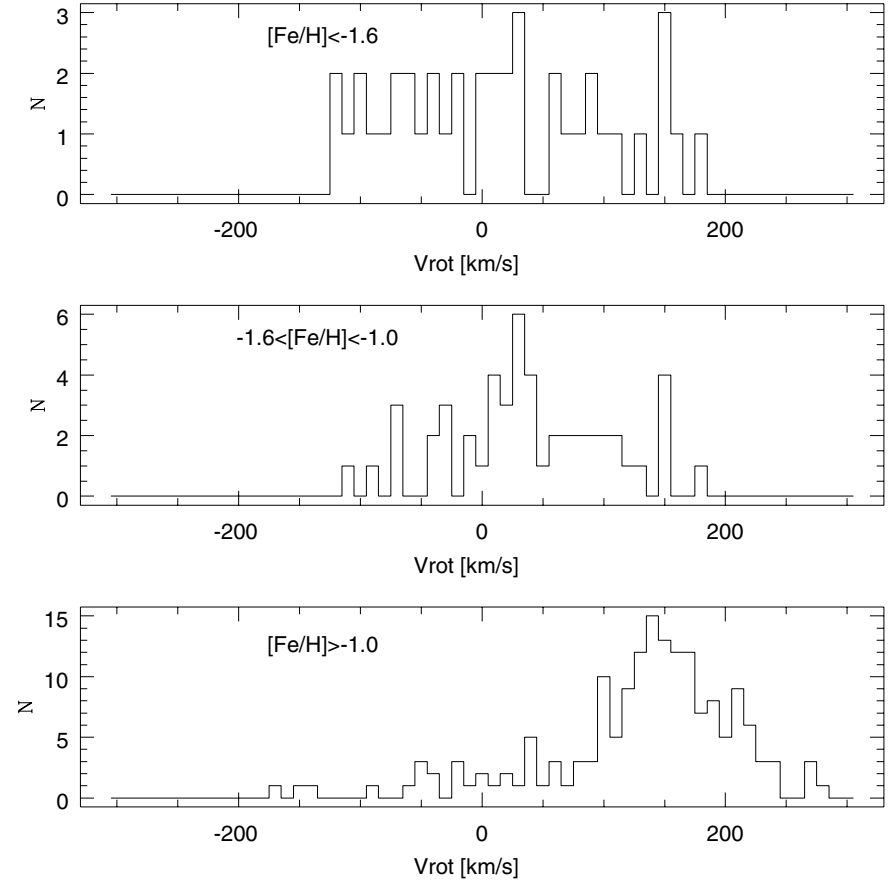

Fig. 7. The same as Fig. 6, but for sample CLLA-Tycho 2.

On the other hand, the asymmetric drift ratio $\langle V\rangle / \sigma_{\mathrm{U}}^{2}=$ -0.007 (CLLA-TYC2+HIP) is very similar to that of the thick disk stars, $\langle V\rangle / \sigma_{U}^{2}=-0.008$. We conclude tentatively that the stars in the $-1.6 \leq[\mathrm{Fe} / \mathrm{H}] \leq-1.0$ metallicity range represent a population of the dynamically hot metal-weak thick disk (MWTD).

\section{Summary and discussion}

We have analyzed the kinematics of 742 nearby metal-poor subdwarf stars from the CLLA catalogue. The subdwarfs were cross-identified with the TYC2+HIP and Tycho 2 Catalogues to find accurate trigonometric parallaxes and proper motions. The accurate Hipparcos parallaxes lead to an upward correction factor of $11 \%$ of the photometric distance scale of CLLA, and it was used to correct the photometric distances of CLLA-Tycho 2 stars.

The present analysis indicates that the solar neighborhood subdwarf stars with $[\mathrm{Fe} / \mathrm{H}]<-1.6$ show halo kinematics characterized by a radially elongated velocity ellipsoid and no significant rotation. At a metallicity range of $[\mathrm{Fe} / \mathrm{H}]>-1$, our samples show disklike kinematics. In the metallicity range $-1.6 \leq[\mathrm{Fe} / \mathrm{H}] \leq-1.0$ we found a significant number of stars with kinematics not of halo stars but that of a dynamically not-metal-weak tail of the thick disk.

Chiba \& Beers (2000) obtained a fraction of $30 \%$ of lowmetallicity stars in their nonkinematically selected solar neighborhood sample with $-1.7<[\mathrm{Fe} / \mathrm{H}] \leq-1.0$, which is consistent with our result of $18 \%$. Chiba \& Yoshii (1998) analyzed the kinematics of red giants and RR Lyrae stars in the solar neighborhood based on Hipparcos data. They found in both red giant and RR Lyrae samples in the range $-1.6<[\mathrm{Fe} / \mathrm{H}] \leq-1.0$, a fraction of $\sim 10 \%$ of stars in a population with a mean velocity $\left\langle V_{\phi}\right\rangle_{\text {disk }}=195 \mathrm{~km} \mathrm{~s}^{-1}$. 
We must try to understand the implications of a significant population of MWTD stars for theories of the formation and evolution of the Galaxy. It should be kept in mind that, although the MWTD population may contribute a large fraction of the local metal-poor stars, the (inner) halo population is probably still dominated by the stars with $[\mathrm{Fe} / \mathrm{H}] \leq-1.6$ within a few kiloparsecs of the Sun. Furthermore, although we have emphasized the possible importance of the MWTD population, it certainly still appears to be a minor constituent of the entire thick disk population (Beers et al. 2002).

If there is indeed a significant fraction of thick disk stars with metal abundance $-1.6 \leq[\mathrm{Fe} / \mathrm{H}] \leq-1$, as we have argued, this finding may have significance for formation scenarios of the Galaxy. An interesting scenario for the origin of an MWTD component may be the merging of satellite galaxies (Searle \& Zinn 1978), which are then accreted by a thin, fast rotating, possibly metal-poor, Galactic disk (Quinn et al. 1993; Wyse 2001). The dynamical heating of the stellar component of this disk in connection with the accretion process produces the thick disk. The kinematics of the halo depends on the dynamics of the merging satellites, whereas the kinematics of the thick disk are determined by the heating of the rotating thin disk. Based on this merging picture of galaxy formation, one might argue that the "shredded satellite" stars retain a kinematic signature distinct from the thick disk part that results from the heated thin disk. The kinematic trace of the destroyed satellite, which is probably the origin of the MWTD stars, would be visible in the mean orbital rotational velocity of stars. Based on a spectroscopic survey of $\sim 2000 \mathrm{~F} / \mathrm{G}$ stars $0.5-5 \mathrm{kpc}$ above the Galactic plane Gilmore et al. (2002) determined a mean rotational velocity lag of the shredded galaxies of $\sim 100 \mathrm{~km} \mathrm{~s}^{-1}$. The actual lag expected from the shredded satellite depends predominantly on the initial orbit and the amount of angular momentum transport in the merger process and is not initially predictable in a specific case (Gilmore et al. 2002).

Acknowledgements. M.I.A. acknowledges support for this work as part of a Ph.D Thesis from a DAAD scholarship.

\section{References}

Beers, T. C., Drilling, J. S., Rossi, S., et al. 2002, AJ, 124, 931

Beers, T. C., \& Sommer-Larsen, J. 1995, ApJS, 96, 175
Carney, B. W., Latham, D. W., Laird, J. B., \& John, B. 1989, AJ, 97, 423

Carney, B. W., Latham, D. W., Laird, J. B., \& Aguilar, L. A. 1994, AJ, 107, 2240 (CLLA)

Carney, B. W., Laird, J. B., Latham, D. W., \& Aguilar, L. A. 1996, AJ, 112,668

Chiba, M., \& Beers, T. C. 2000, AJ, 119, 2843

Chiba, M., \& Yoshii, Y. 1998, AJ, 115, 168

Delhaye, J. 1965, in Galactic Structure, Stars and Stellar Systems 5, 61

European Space Agency (ESA) 1997, The Hipparcos and Tycho Catalogues, ESA SP-1200 (Noordwijk: ESA)

Freeman, K. C. 1987, ARA\&A, 25, 603

Fuchs, B., Jahreiß, H., \& Wielen, R. 1998, Ap\&SS, 265, 175 (FJW)

Gilmore, G., \& Wyse, R. F. G. 1985, AJ, 90, 2015

Gilmore, G., Wyse, R. F. G., \& Kuijken, K. 1989, ARA\&A, 27, 555

Gilmore, G., Wyse, R. F. G., \& Norris, J. N. 2002, AJ, 574, L39

Høg, E., Fabricius, C., Makarov, V. V., et al. 2000, A\&A, 355, 367

Jahreiß, H., Fuchs, B., \& Wielen, R. 1997, in Hipparcos-Venice '97, ed. M.A.C. Perryman, P.L. Bernacca, ESA SP-402, 587

Laird, J. B., Rupen, M. P., Carney, B. W., \& Latham, D. W. 1988, AJ, 96, 1908

Layden, A. C., Hanson, R. B., Hawley, S. L., Klemola, A. R., \& Hanley, C. J. 1996, AJ, 112, 2110

Martin, J. C., \& Morrison, H. L. 1998, AJ, 116, 172

Morrison, H. L., Flynn, C., \& Freeman, K. C. 1990, AJ, 100, 1191 (MFF)

Nissen, P. E., \& Schuster, W. J. 1991, A\&A, 251, 457

Press, W. H., Flannery, B. P., Teukolsky, T. A., \& Vetterling, W. T. 1992, Numerical Recipes in Fortran (Cambridge Univ. Press)

Quinn, P. J., Hernquist, L., \& Fullagar, D. P. 1993, ApJ, 403, 74

Reid, I. N. 1998, AJ, 115, 204

Ryan, S. G., \& Norris, J. E. 1991, AJ, 101, 1835

Sachs, L. 1988, Statistische Methoden (Berlin: Springer Verlag)

Searle, L., \& Zinn, R. 1978, ApJ, 225, 357

Sommer-Larsen, J., \& Zhen, C. 1990, MNRAS, 242, 10

Wielen, R. 1986, Transactions Intern. Astron. Union 19B, 93

Wielen, R., Schwan, H., Dettbarn, C., et al. 2001, Astrometric Catalogue TYC2+HIP Derived from a Combination of the HIPPARCOS Catalogue with the Proper Motions Given in the TYCHO-2 Catalogue, Veröff. Astron. Rechen-Inst, Heidelberg No. 39

Wyse, R. F. G. 2001, in Galaxy Disks and Disk Galaxies, ed. J. G. Funes \& E. M. Corsini, ASP Conf. Ser., 230, 71 